\title{
Genome-Wide Characterisation of Gene Expression in Rice Leaf Blades at $25^{\circ} \mathrm{C}$ and $30^{\circ} \mathrm{C}$
}

\author{
Zhi-guo E, ${ }^{1}$ Lei Wang, ${ }^{1}$ Ryan Qin, ${ }^{2}$ Haihong Shen, ${ }^{3}$ and Jianhua Zhou ${ }^{4}$ \\ ${ }^{1}$ China National Rice Research Institute, No. 359, Tiyuchang Road, Hangzhou 310006, China \\ ${ }^{2}$ iBioinfo Group, Lexington, MA 02421, USA \\ ${ }^{3}$ School of Life Science, Gwangju Institute of Science and Technology, Gwangju 500-712, Republic of Korea \\ ${ }^{4}$ Nantong University, Nantong 226001, China \\ Correspondence should be addressed to Jianhua Zhou; jianhua55@msn.com
}

Received 5 August 2013; Accepted 30 September 2013; Published 29 January 2014

Academic Editors: B. Shen, J. Wang, and J. Wang

Copyright ( 2014 Zhi-guo E et al. This is an open access article distributed under the Creative Commons Attribution License, which permits unrestricted use, distribution, and reproduction in any medium, provided the original work is properly cited.

\begin{abstract}
Rice growth is greatly affected by temperature. To examine how temperature influences gene expression in rice on a genomewide basis, we utilised recently compiled next-generation sequencing datasets and characterised a number of RNA-sequence transcriptome samples in rice seedling leaf blades at $25^{\circ} \mathrm{C}$ and $30^{\circ} \mathrm{C}$. Our analysis indicated that $50.4 \%$ of all genes in the rice genome $(28,296 / 56,143)$ were expressed in rice samples grown at $25^{\circ} \mathrm{C}$, whereas slightly fewer genes $(50.2 \% ; 28,189 / 56,143)$ were expressed in rice leaf blades grown at $30^{\circ} \mathrm{C}$. Among the genes that were expressed, approximately $3 \%$ were highly expressed, whereas approximately $65 \%$ had low levels of expression. Further examination demonstrated that 821 genes had a twofold or higher increase in expression and that 553 genes had a twofold or greater decrease in expression at $25^{\circ} \mathrm{C}$. Gene ontology (GO) and Kyoto Encyclopedia of Genes and Genomes (KEGG) analyses suggested that the ribosome pathway and multiple metabolic pathways were upregulated at $25^{\circ} \mathrm{C}$. Based on these results, we deduced that gene expression at both transcriptional and translational levels was stimulated at $25^{\circ} \mathrm{C}$, perhaps in response to a suboptimal temperature condition. Finally, we observed that temperature markedly regulates several super-families of transcription factors, including bZIP, MYB, and WRKY.
\end{abstract}

\section{Introduction}

Rice (Oryza sativa L.) includes two major subspecies, indica and japonica. Owing to its importance in food security, extensive studies utilising genetic manipulation, improved cultivation and crossing of subspecies have been conducted in rice during recent decades to improve quality and yield. Like all other plants, rice constantly experiences environmental changes and hostile abiotic stress conditions, such as drought, cold, pollution due to heavy metals and salinity, in addition to biotic stresses, such as viral infection. To minimise abiotic and/or biotic stress-induced damage, plants have developed adaptations and stress tolerance during evolution through the regulation of gene expression and changes in cellular processes. Two major pathways, abscisic acid (ABA)-dependent and ABA-independent pathways, have been extensively studied in plants in response to biotic and/or abiotic stresses [13]. ABA is a hormone produced during metabolic reactions.
In response to abiotic stress, the ABA-dependent pathway induces the expression of many stress-related genes by regulating the activities of transcription factors. Among many transcription factors, several super-families, including basic leucine zipper (bZIP) [4-6], MYB [7, 8], and WRKY [9-11], have been shown to play critical roles in the regulation of stress response genes in rice.

bZIP proteins include a family of transcriptional regulators that are exclusively present in eukaryotes. Furthermore, they characteristically harbour a bZIP domain composed of two structural features: a DNA-binding basic region and the leucine-zipper dimerisation region. They have been shown to regulate diverse plant-specific phenomena, including seed maturation and germination, flower induction and development, photomorphogenesis and stress and hormone signalling. There are approximately 90 bZIP transcription factor-encoding genes in the rice genome [5]. 
The MYB gene family includes at least 155 members that have been identified by a genome-wide analysis and represents one of the richest groups of transcription factors in rice. MYB proteins are characterised by a highly conserved MYB DNA-binding domain and can be classified into four major groups, 1R-MYB, 2R-MYB, 3R-MYB, and 4R-MYB, on the basis of the number and position of MYB repeats. MYB transcription factors are involved in plant development, secondary metabolism, hormone signal transduction, disease resistance, and abiotic stress tolerance [12].

WRKY genes encode transcription factors with a WRKY domain that belongs to zinc-finger proteins. WRKY proteins contain one or two conserved WRKY domains, which are encoded by approximately $60 \mathrm{~N}$-terminal amino acid residues with a WRKYGQ(K/E)K sequence, followed by a $\mathrm{C} 2 \mathrm{H} 2$ or $\mathrm{C} 2 \mathrm{HC}$ zinc-finger motif. An exhaustive search for WRKY genes using HMMER and a hidden Markov model resulted in the identification of 98 and 102 WRKY genes in $O$. japonica and O. indica rice, respectively. WRKY genes play important roles in disease resistance, responses to salicylic and jasmonic acid, seed development and germination, senescence, abiotic stress responses and $\mathrm{ABA}$ responses in rice [13].

Despite all this knowledge, the mechanisms that regulate gene expression in rice are not completely understood. To investigate how external factors, such as temperature, affect rice development and growth through the regulation of gene expression, we searched the available transcriptome databases. We identified two transcriptome RNA-sequence (RNA-Seq) datasets of high quality from rice seedling leaf blades leaf blades grown at $25^{\circ} \mathrm{C}$ or $30^{\circ} \mathrm{C}$. We found that the expression of more than 1300 genes in rice showed a twofold or higher difference between leaf blades that were grown at $25^{\circ} \mathrm{C}$ compared with those grown at $30^{\circ} \mathrm{C}$. Gene ontology (GO) and Kyoto Encyclopedia of Genes and Genomes (KEGG) analyses showed that transcription of many abiotic stress genes and genes involved in ribosome biogenesis were induced at $25^{\circ} \mathrm{C}$, indicating that rice grown at $25^{\circ} \mathrm{C}$ has more active transcription and translation than rice grown at $30^{\circ} \mathrm{C}$. Furthermore, we found that among the transcription factor super-families, bZIP, MYB, NAC, and WRKY were significantly regulated in rice at $25^{\circ} \mathrm{C}$. Our studies provide useful information on the rice transcriptome in response to suboptimal temperatures.

\section{Materials and Methods}

2.1. Transcriptome Sequencing Datasets of Rice Seedling Leaf Blades. Two publicly available RNA-Seq datasets using deepsequencing of rice seedling leaf blades were downloaded from Gene Expression Omnibus (GEO) under the accession number GSE42096 (http://www.ncbi.nlm.nih.gov/geo/ query/acc.cgi?acc=GSE42096) and used for primary analyses. The leaf blades analysed were obtained from wild-type seedlings grown at $30^{\circ} \mathrm{C}$ or $25^{\circ} \mathrm{C}$. For each dataset, RNA-Seq was conducted by paired-end approaches using an Illumina HiSeq 2000 instrument. The read length was $90 \mathrm{bp}$.
2.2. Sequencing Analysis. Sequence alignment between the transcriptome reads was conducted and reads were checked for quality and mapped to the reference genome sequences by Bowtie 2 using the parameters "end-to-end" and "verysensitive". The reference genome, transcript annotation, and GO datasets were downloaded from MSU Rice Genome Annotation Project, release 7. The number of reads for a gene was designated as reads per $\mathrm{kb}$ per million total reads (RPKM) after normalisation to the number of mapped genome locations. KEGG gene classifications were downloaded from its database.

2.3. Statistical Analyses. To determine whether expression was differentially regulated under different temperatures $\left(25^{\circ} \mathrm{C}\right.$ versus $\left.30^{\circ} \mathrm{C}\right)$, we conducted statistical analyses based on the fold-changes in gene expression by adding median counts as a pseudocount. Pathway analyses were based on the binomial probability of observing a number of gene changes in a given pathway. Differences were considered statistically significant when the $P$ value was $<0.05$.

\section{Results}

3.1. RNA-Seq Datasets of the Transcriptome from Rice. To accurately determine rice gene expression profiles, we took advantage of recent advances in deep-sequencing technologies. Many RNA-Seq datasets of the transcriptome of rice and other plants are publicly available. Using these datasets, we identified transcriptome sequencing libraries of two rice samples in a single GEO dataset, GSE42096, generated by the Chinese Academy of Sciences and the National Centre for Plant Gene Research (http://www.ncbi.nlm.nih.gov/geo/ query/acc.cgi?acc=GSE42096). Profiling of these transcriptome RNAs by high-throughput sequencing was conducted by both single-end and paired-end approaches using an Illumina HiSeq 2000 platform. Paired-end sequencing provided 90 basepairs (bp) per read from each end. RNA-Seq of the transcriptome for each sample generated approximately 25 million reads. Sequence analysis indicated that the datasets were of exceptionally high quality with very low background noise (Supplementary Figure 1 available online on http://dx.doi.org/10.1155/2014/917292).

3.2. Gene Expression in Rice at $25^{\circ} \mathrm{C}$ and $30^{\circ} \mathrm{C}$. To characterize expressed transcripts, a reference genome dataset was required. We searched existing databases and found that the MSU Rice Genome Annotation Project on O. sativa japonica, released on 31st Oct 2011, is the most complete rice genome database available with more than 56,143 annotated genes, slightly more than the 55986 reported by Kawahara et al. [14]. Therefore, we used this database as our reference for analysing gene expression in the two leaf blade transcriptome datasets. We used Bowtie 2 to align and map the transcriptome reads to rice genome. On the basis of our analysis, we calculated the number of reads as reads per billion (RPB) for each mRNA in two samples. We normalised RPB by read per kbp and RPKM. We found that at $25^{\circ} \mathrm{C}$ there were 19,766 annotated genes with $>1$ RPKM read, whereas at $30^{\circ} \mathrm{C}$ 
TABLE 1: Top ranked pathways that are regulated (1374 genes) at $25^{\circ} \mathrm{C}$ by GO analysis.

\begin{tabular}{|c|c|c|c|c|c|c|}
\hline Rank & Pathway & Pathway annotation & Pathway size & Observed & Ratio & $P$ value \\
\hline 1 & Process: metabolic process & rice:GO:0008152 & 7390 & 391 & 0.05 & $4.3 E-52$ \\
\hline 2 & Process: response to stress & rice:GO:0006950 & 3620 & 241 & 0.07 & $1.9 E-46$ \\
\hline 3 & Process: response to abiotic stimulus & rice:GO:0009628 & 2195 & 177 & 0.08 & $1.5 E-44$ \\
\hline 4 & Process: cellular process & rice:GO:0009987 & 7325 & 365 & 0.05 & $3.3 E-42$ \\
\hline 5 & Function: catalytic activity & rice:GO:0003824 & 3688 & 227 & 0.06 & $2.7 E-38$ \\
\hline 6 & Component: membrane & rice:GO:0016020 & 3728 & 220 & 0.06 & $2.1 E-34$ \\
\hline 7 & Process: biosynthetic process & rice:GO:0009058 & 4673 & 250 & 0.05 & $1.6 E-32$ \\
\hline 8 & Process: response to endogenous stimulus & rice:GO:0009719 & 1490 & 118 & 0.08 & $4.9 E-29$ \\
\hline 9 & Component: cytosol & rice:GO:0005829 & 2289 & 151 & 0.07 & $2.1 E-28$ \\
\hline 10 & Process: response to biotic stimulus & rice:GO:0009607 & 1081 & 96 & 0.09 & $1.9 E-27$ \\
\hline 11 & Component: ribosome & rice:GO:0005840 & 481 & 61 & 0.13 & $9.5 E-26$ \\
\hline 12 & Function: structural molecule activity & rice:GO:0005198 & 518 & 63 & 0.12 & $1.5 E-25$ \\
\hline
\end{tabular}

the number of genes that had at least 1 RPKM was 19,350. The distribution of expressed genes with different RPKM levels was similar between $25^{\circ} \mathrm{C}$ and $30^{\circ} \mathrm{C}$ (Supplementary Figure 2). Approximately 30 genes had extremely high expression levels (>1000 RPKM), 3\% of annotated genes (550-600) were highly expressed ( $>100$ RPKM) and more than 33\% of expressed genes (6900) were expressed at only a modest level (10-100 RPKM). Of the genes that were expressed, $64 \%$ $(12,000)$ had fewer than 9 RPKM, suggesting that most genes were expressed at a low level. Expression of the other 36,000 genes was not detectable.

3.3. Differential Gene Expression in Rice between $30^{\circ} \mathrm{C}$ and $25^{\circ} \mathrm{C}$. We then compared the genes with varied expression in rice growing at $30^{\circ} \mathrm{C}$ and $25^{\circ} \mathrm{C}$. We calculated ratios between the number of reads at $30^{\circ} \mathrm{C}$ and the number of reads at $25^{\circ} \mathrm{C}$. As shown in Supplementary Figure 3, the left part of the histogram shows the number of genes with an increased expression at $25^{\circ} \mathrm{C}$. We found that the expression of 257 genes was upregulated more than threefold in rice grown at $25^{\circ} \mathrm{C}$, whereas expression of 173 genes was downregulated more than threefold at $25^{\circ} \mathrm{C}$. Moreover, there were more genes that were upregulated (564) than downregulated $(380)$ at $25^{\circ} \mathrm{C}$, with expression level changes between twofold and threefold. Among approximately 2712 genes with a 1.5-2.0-fold change in expression, 1617 genes were upregulated and 1095 genes (1.5-2.0-fold) were downregulated. Our results indicate that more genes were upregulated at $25^{\circ} \mathrm{C}$ than at $30^{\circ} \mathrm{C}$, suggesting that at $25^{\circ} \mathrm{C}$, rice plants need to respond to a suboptimal lower temperature by altering gene transcription.

3.4. GO Analysis of Genes That Are Upregulated and Downregulated. To examine the mechanisms of the molecular and cellular responses to a suboptimal lower temperature of $25^{\circ} \mathrm{C}$, we performed GO and KEGG analysis in genes of the rice transcriptome that had an twofold or higher change in expression between $25^{\circ} \mathrm{C}$ and $30^{\circ} \mathrm{C}$. In total, we found that the expression of 821 genes was upregulated by twofold or higher at $25^{\circ} \mathrm{C}$, whereas 553 genes showed downregulated expression at $25^{\circ} \mathrm{C}$. Among the 1374 genes that were either upregulated or downregulated, GO analysis indicated that 4 of the 10 top-ranked GO categories were stress related, with $17.54 \%$ of genes (241/1374) related to "response to stress" (ranked at no. 2 with $P=1.88 E-46$ ), $12.88 \%$ of genes (177/1374) related to "response to abiotic stimulus" (ranked at no. 3 with $P=1.55 E-44), 8.59 \%$ of genes (118/1374) related to "response to endogenous stimulus" (ranked at no. 8 with $P=4.90 E-29)$ and $6.99 \%$ of genes $(96 / 1374)$ related to "response to biotic stimulus" (ranked at no. 10 with $P=1.91 E-27)$, suggesting that $25^{\circ} \mathrm{C}$ could be considered as a cold-stress condition (Table 1). Other high-ranked GO categories included membrane processes (ranked no. 1) and metabolic processes and ribosome (ranked no. 11), indicating that metabolism and protein translation are perhaps also upregulated at $25^{\circ} \mathrm{C}$.

3.5. KEGG Pathway Analysis of Upregulated and Downregulated Genes. To further characterise the pathways that are involved in temperature-induced stress responses, we performed KEGG pathway analysis. We observed that the expression of genes that are involved in ribosome biogenesis was significantly upregulated at $25^{\circ} \mathrm{C}$, with an adjusted $P$ value of $5.2 E-29$ (Table 2). In addition, 57 of the potential 362 transcripts that had been annotated in the ribosome pathway had a twofold or higher increase in expression at $25^{\circ} \mathrm{C}$ compared with $30^{\circ} \mathrm{C}$. These upregulated transcripts represent $15.7 \%$ of the genes in the ribosome pathway. In contrast, none of the 362 transcripts showed decreased expression. Taken together, these results strongly suggested that both transcription and translation were more active at $25^{\circ} \mathrm{C}$ than $30^{\circ} \mathrm{C}$. Other major pathways showing significant changes in expression on KEGG analysis included metabolic and biosynthesis pathways. Of note, $P$ values for pathways that were upregulated were markedly more significant than pathways that were downregulated.

3.6. Expression Analysis of bZIP, WRKY and MYB Transcription Factors. The relative growth rate (RGR) in rice is influenced by temperature, with an optimal growth rate at $30^{\circ} \mathrm{C}$ [15]. Rice also has a stress response mechanism that 
TABLE 2: Top ranked pathways that are regulated (1374 genes) at $25^{\circ} \mathrm{C}$ by KEGG analysis.

\begin{tabular}{|c|c|c|c|c|c|c|}
\hline Rank & Pathway & Pathway annotation & Pathway size & Observed & Ratio & $P$ value \\
\hline 1 & Ribosome & rice:osa 03010 & 362 & 57 & 0.16 & $5.2 E-29$ \\
\hline 2 & Metabolic pathways & rice:osa01100 & 1565 & 119 & 0.08 & $1.0 E-27$ \\
\hline 3 & Biosynthesis of secondary metabolites & rice:osa01110 & 745 & 65 & 0.09 & $1.3 E-18$ \\
\hline 4 & Starch and sucrose metabolism & rice:osa00500 & 130 & 20 & 0.15 & $5.9 E-11$ \\
\hline 5 & Alpha-Linolenic acid metabolism & rice:osa00592 & 34 & 9 & 0.26 & $8.7 E-08$ \\
\hline 6 & Glyoxylate and dicarboxylate metabolism & rice:osa00630 & 62 & 11 & 0.18 & $2.6 E-7$ \\
\hline 7 & Carbon fixation in photosynthetic organisms & rice:osa00710 & 85 & 12 & 0.14 & $9.6 E-07$ \\
\hline 8 & Diterpenoid biosynthesis & rice:osa00904 & 24 & 7 & 0.29 & $1.2 E-06$ \\
\hline 9 & Photosynthesis & rice:osa00195 & 148 & 15 & 0.10 & $3.2 E-06$ \\
\hline 10 & Plant hormone signal transduction & rice:osa04075 & 150 & 14 & 0.09 & $1.6 E-05$ \\
\hline 11 & Biosynthesis of unsaturated fatty acids & rice:osa01040 & 44 & 7 & 0.16 & $8.0 E-05$ \\
\hline 12 & Phenylpropanoid biosynthesis & rice:osa00940 & 94 & 9 & 0.10 & $4.0 E-04$ \\
\hline
\end{tabular}

TABLE 3: Top ranked transcription factors that are regulated in rice by temperature.

\begin{tabular}{|c|c|c|c|c|c|c|}
\hline Rank & Pathway & Pathway annotation & Pathway size & Observed & Ratio & $P$ value \\
\hline 1 & Rice transcription factor: WRKY & rice:TF:WRKY & 107 & 15 & 0.14 & $4.9 E-08$ \\
\hline 2 & Rice transcription factor: NAC & rice:TF:NAC & 124 & 12 & 0.096 & $4.5 E-05$ \\
\hline 3 & Rice transcription factor: AP2-EREBP & rice:TF:AP2-EREBP & 169 & 14 & 0.082 & $5.9 E-05$ \\
\hline 4 & Rice transcription factor: orphans & rice:TF:orphans & 85 & 9 & 0.105 & 0.00019 \\
\hline 5 & Rice transcription factor: bZIP & rice:TF:bZIP & 95 & 9 & 0.095 & 0.00043 \\
\hline 6 & Rice transcription factor: MYB & rice:TF:MYB & 128 & 10 & 0.078 & 0.00092 \\
\hline 7 & Rice transcription factor: tify & rice:TF:tify & 21 & 4 & 0.190 & 0.00140 \\
\hline 8 & Rice transcription factor: MYB-related & rice:TF:MYB-related & 100 & 7 & 0.07 & 0.00835 \\
\hline 9 & Rice transcription factor: $\mathrm{C} 2 \mathrm{H} 2$ & rice:TF:C2H2 & 104 & 7 & 0.067 & 0.01004 \\
\hline 10 & Rice transcription factor: pseudo ARR-B & rice:TF:pseudo_ARR-B & 9 & 2 & 0.222 & 0.01811 \\
\hline 11 & Rice transcription factor: G2-like & rice:TF:G2-like & 48 & 4 & 0.083 & 0.02342 \\
\hline 12 & Rice transcription factor: WRKY & rice:TF:WRKY & 107 & 15 & 0.140 & 4.95634 \\
\hline
\end{tabular}

is triggered in response to lower temperatures. As described above, transcription is more active at $25^{\circ} \mathrm{C}$ than at $30^{\circ} \mathrm{C}$, indicating that gene expression is stimulated at $25^{\circ} \mathrm{C}$. To characterise transcription factors that may be involved in the regulation of gene expression in rice growth at different temperatures and to understand how rice responds to the suboptimal temperature of $25^{\circ} \mathrm{C}$, we analysed transcription factor families in rice (Supplementary Table S1), including the expression distribution patterns of the bZIP, MYB, WRKY, and HLH transcription factor super-families (Table 3, Supplementary Table S1 and Figure 1). Approximately 9.5\% of bZIP (9/95), 14.1\% of WRKY (15/107), $7.8 \%$ of MYB $(12 / 128)$ and $3.5 \%$ of HLH (5/145) transcription factors had a twofold or higher change in expression. In contrast, a random calculation suggested that $<2.57 \%(1374 / 56,143)$ of the genes should be upregulated or downregulated by twofold or more. Therefore, we conclude that expression of bZIP, WRKY, and MYB super-families was significantly regulated by temperature, with $P$ values of $4.96 E-08$ (WRKY), $4.3 E-04$ (bZIP) and $0.008(\mathrm{MYB})$ between $25^{\circ} \mathrm{C}$ and $30^{\circ} \mathrm{C}$. These data are consistent with previous reports $[16,17]$ showing that these transcription factor super-families are upregulated or downregulated under colder or warmer temperatures. Other highly regulated transcription factor super-families include
NAC and AP2-EREBP. In contrast, other transcription factors such as bHLH and HB did not exhibit significant changes in expression.

\section{Discussion}

Like all plants, rice has to endure constant environmental changes. Among many factors, temperature has been shown to greatly influence rice growth. Rice can grow at a range of temperature, from as low as $12^{\circ} \mathrm{C}$ to as high as $40^{\circ} \mathrm{C}$, but its optimal growth temperature is $30^{\circ} \mathrm{C}$ or warmer [15]. Unlike mammals, which have a constant body temperature, rice grows at temperatures that fluctuate daily between night and day. One of the mechanisms by which rice can adjust to temperature changes is through regulation of gene expression. Extensive studies have been conducted in rice to analyse the molecular basis of adaptation to both warmer temperatures and cold-stress conditions [3, 18-24]. However, global surveys of temperature-dependent changes in rice gene expression, particularly studies using next generation sequencing technology, are not extensive.

Because the rapid development and reduced cost of both next-generation sequencing and microarray technology, researchers have regularly deposited RNA-Seq datasets for 


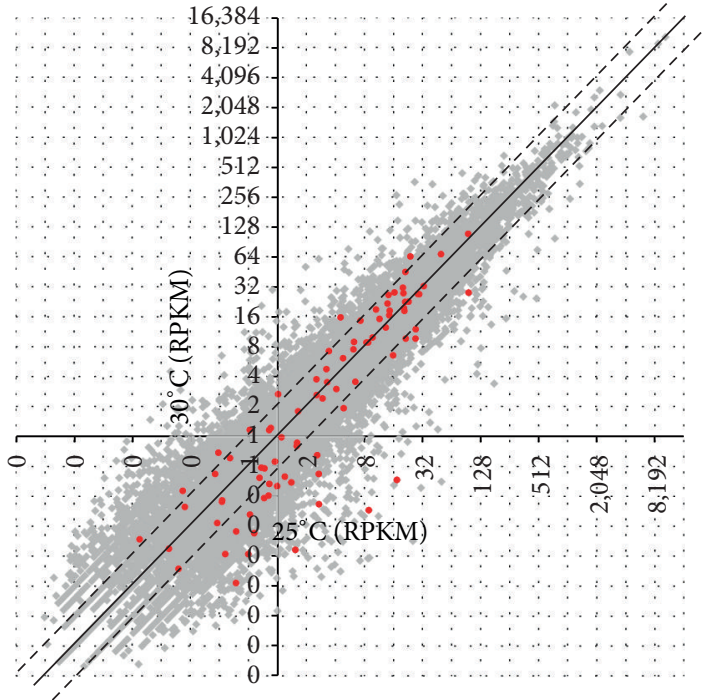

- WRKY

- $25^{\circ} \mathrm{C}$ versus $30^{\circ} \mathrm{C}$ (RPKM)

(a)

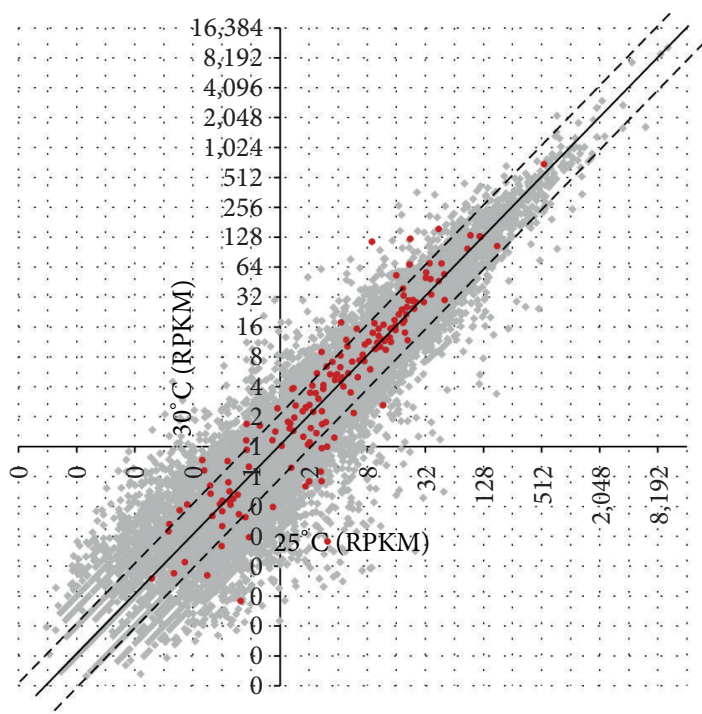

- MYB

* $25^{\circ} \mathrm{C}$ versus $30^{\circ} \mathrm{C}$ (RPKM)

(c)

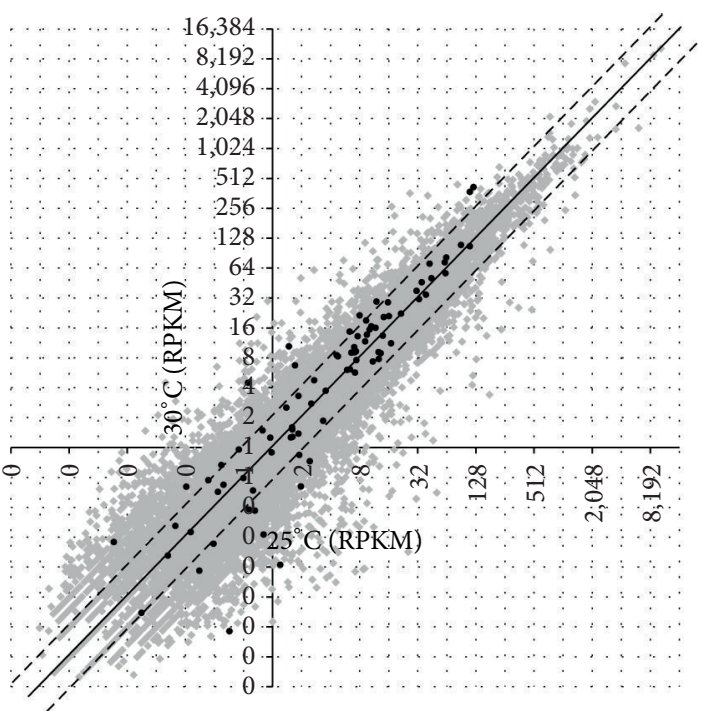

- bZIP

\ $25^{\circ} \mathrm{C}$ versus $30^{\circ} \mathrm{C}$ (RPKM)

(b)

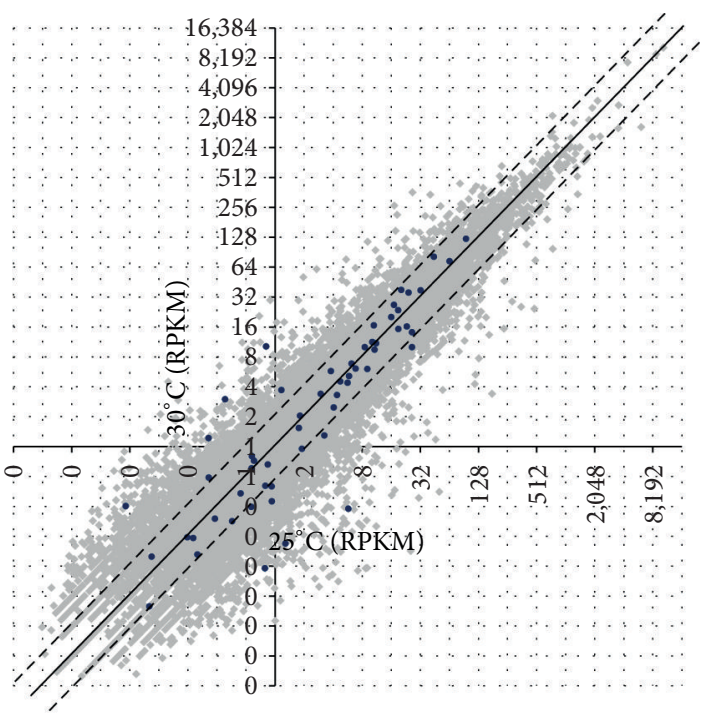

- HLH

* $25^{\circ} \mathrm{C}$ versus $30^{\circ} \mathrm{C}(\mathrm{RPKM})$

(d)

Figure 1: A scatter plot of gene expression values (per kb per million total reads, RPKM) in log 2 scale for the $25^{\circ} \mathrm{C}$ versus $30^{\circ} \mathrm{C}$ datasets. Grey dots indicate each gene expressed in the two samples. Coloured dots indicate a specific group of genes. Two diagonal dashed lines indicate twofold changes (increases and decreases).

many organisms, including rice, in public domains. For example, several recent papers reported gene expression and splicing in rice using NGS and bioinformatics analysis [25-28]. To investigate how temperature may influence rice growth by affecting gene expression, we searched GEO databases (http://www.ncbi.nlm.nih.gov/geo/). We identified four sets of RNA-Seq transcriptome data from rice (GSE42096, GSE39307, GSE30490, and GSE27240) that were of a high quality. However, among these datasets, only four samples in the GSE42096 dataset, which includes wild-type and TOG1 mutant rice leaf blades, were related to different temperatures $\left(25^{\circ} \mathrm{C}\right.$ and $\left.30^{\circ} \mathrm{C}\right)$. Because mutant TOG1 has not yet been fully described in a public domain, we analysed only two wild-type rice leaf blade samples that grew at both $25^{\circ} \mathrm{C}$ and $30^{\circ} \mathrm{C}$. We realised that the number of samples (two) was limited, but because the quality of these RNA-Seq datasets was exceptionally high, with more than $25 \mathrm{M}$ reads each sample, we believe that our analysis will provide useful 
information on gene expression in rice to complement similar studies.

Gene expression is regulated at multiple levels. The most fundamental regulatory mechanisms that control the amount of proteins produced are transcription and translation. The cis-sequences in a gene, in particular the promoter, and transcription factors dictate how much RNA is transcribed from a gene, whereas ribosomes are directly related to the activities of protein translation. In our investigation, we demonstrated that the number of the genes that are expressed at $30^{\circ} \mathrm{C}(17,356)$ is similar to that at $25^{\circ} \mathrm{C}(17,966)$, indicating that it is necessary for only $1 / 3$ of rice genes to be expressed to maintain growth under a given condition. Similarly, we observed that about $3 \%$ of genes were highly expressed at both $30^{\circ} \mathrm{C}$ and $25^{\circ} \mathrm{C}$. The rest of the expressed genes had either a modest or a low expression level or no detectable expression.

However, the difference lies in the expression levels of specific genes between samples at $25^{\circ} \mathrm{C}$ and $30^{\circ} \mathrm{C}$. We showed that 3986 genes had either increased or decreased expression levels of 1.50-fold or higher. Among these 3986 genes, 1374 had a twofold or higher increase (821) or decrease (553) when the temperature dropped from $30^{\circ} \mathrm{C}$ to $25^{\circ} \mathrm{C}$, indicating perhaps that rice at $25^{\circ} \mathrm{C}$ has more active transcription. Consistent with this notion, by GO and KEGG analyses, we found that a significant number of genes in the ribosome pathways were upregulated at $25^{\circ} \mathrm{C}$, suggesting that translation may be also more robust at $25^{\circ} \mathrm{C}$. Considering previous reports that rice has a better growth rate at $30^{\circ} \mathrm{C}$, or at least at temperatures warmer than $25^{\circ} \mathrm{C}$ [15], the more active transcription and translation at $25^{\circ} \mathrm{C}$ can be explained by the response of rice to a colder temperature. In fact, we observed in our GO analysis that 4 of the 12 top-ranked pathway categories were related to stress response. We deduced from our results that although $30^{\circ} \mathrm{C}$ is an optimal temperature for rice growth, transcription and translation for many genes are triggered at $25^{\circ} \mathrm{C}$ and this temperature $\left(25^{\circ} \mathrm{C}\right)$ may be minimally sufficient to trigger the cold stress-response.

To further examine the molecular basis of the differential expression of rice genes between $25^{\circ} \mathrm{C}$ and $30^{\circ} \mathrm{C}$, we examined rice transcription factor families (Figure 1, Table 3, Supplementary Table S1). In contrast to the WRKY super-family, bZIP and MYB transcription factor super-families had more downregulated than upregulated genes (1.5-fold cutoff) at $25^{\circ} \mathrm{C}$. Although we were unable to draw definitive conclusions from this analysis, our results suggest that the WRKY superfamily plays a positive role in the response to lower temperatures, whereas both bZIP and MYB super-families may have a negative impact on gene expression at $25^{\circ} \mathrm{C}$, in agreement with a previous report that bZIP transcription factors, such as ZIP52, are negative regulators of cold stress [5]. In addition, we also showed that many transcription factors, including bHLH and $\mathrm{HB}$, are not significantly regulated between $30^{\circ} \mathrm{C}$ and $25^{\circ} \mathrm{C}$.

\section{Conclusion}

We concluded that only a small percentage of genes (3\%) have a high expression level whereas more than $60 \%$ of genes have a very low expression level in rice. Both transcription and translation are more active at $25^{\circ} \mathrm{C}$ than at $30^{\circ} \mathrm{C}$. Expression of bZIP, WRKY, and MYB is significantly regulated at $25^{\circ} \mathrm{C}$.

\section{Conflict of Interests}

The authors declare that there is no conflict of interests regarding the publication of this article.

\section{Acknowledgments}

This research was supported by the special fund for Agro-Scientific Research in the Public Interest (201203029, 201203031), the Special Foundation for Basic Research and the Development of Central Level Scientific Research Institutes in China (2012RG006) (ZGE, LW), and the Priority Academic Program Development of Jiangsu Higher Education Institution (PAPD) (JZ).

\section{References}

[1] Y. Fujita, M. Fujita, K. Shinozaki, and K. Yamaguchi-Shinozaki, "ABA-mediated transcriptional regulation in response to osmotic stress in plants," Journal of Plant Research, vol. 124, no. 4, pp. 509-525, 2011.

[2] S. C. Lee and S. Luan, "ABA signal transduction at the crossroad of biotic and abiotic stress responses," Plant, Cell and Environment, vol. 35, no. 1, pp. 53-60, 2012.

[3] G. T. Huang, S. L. Ma, L. P. Bai et al., "Signal transduction during cold, salt, and drought stresses in plants," Molecular Biology Reports, vol. 39, no. 2, pp. 969-987, 2012.

[4] H. Takahashi, T. Kawakatsu, Y. Wakasa, S. Hayashi, and F. Takaiwa, "A rice transmembrane bZIP transcription factor, OsbZIP39, regulates the endoplasmic reticulum stress response," Plant and Cell Physiology, vol. 53, no. 1, pp. 144-153, 2012.

[5] C. Liu, Y. Wu, and X. Wang, "bZIP transcription factor OsbZIP52/RISBZ5: a potential negative regulator of cold and drought stress response in rice," Planta, vol. 235, no. 6, pp. 11571169, 2012.

[6] M. A. Hossain, Y. Lee, J. I. Cho et al., “The bZIP transcription factor OsABF1 is an ABA responsive element binding factor that enhances abiotic stress signaling in rice," Plant Molecular Biology, vol. 72, no. 4, pp. 557-566, 2010.

[7] X. Dai, Y. Xu, Q. Ma et al., "Overexpression of an R1R2R3 MYB gene, OsMYB3R-2, increases tolerance to freezing, drought, and salt stress in transgenic Arabidopsis," Plant Physiology, vol. 143, no. 4, pp. 1739-1751, 2007.

[8] L. Zhang, G. Zhao, J. Jia, X. Liu, and X. Kong, "Molecular characterization of 60 isolated wheat MYB genes and analysis of their expression during abiotic stress," Journal of Experimental Botany, vol. 63, no. 1, pp. 203-214, 2012.

[9] P. Agarwal, M. P. Reddy, and J. Chikara, "WRKY: its structure, evolutionary relationship, DNA-binding selectivity, role in stress tolerance and development of plants," Molecular Biology Reports, vol. 38, no. 6, pp. 3883-3896, 2011.

[10] H. Chen, Z. Lai, J. Shi, Y. Xiao, Z. Chen, and X. Xu, "Roles of arabidopsis WRKY18, WRKY40 and WRKY60 transcription factors in plant responses to abscisic acid and abiotic stress," BMC Plant Biology, vol. 10, article 281, 2010. 
[11] R. Sharma, D. De Vleesschauwer, M. K. Sharma, and P. C. Ronald, "Recent advances in dissecting stress-regulatory crosstalk in rice," Molecular Plant, vol. 6, pp. 250-260, 2013.

[12] A. Nijhawan, M. Jain, A. K. Tyagi, and J. P. Khurana, "Genomic survey and gene expression analysis of the basic leucine zipper transcription factor family in rice," Plant Physiology, vol. 146, no. 2, pp. 333-350, 2008.

[13] C. A. Ross, Y. Liu, and Q. J. Shen, “The WRKY gene family in rice (Oryza sativa)," Journal of Integrative Plant Biology, vol. 49, no. 6, pp. 827-842, 2007.

[14] Y. Kawahara, M. de la Bastide, J. P. Hamilton et al., "Improvement of the Oryza sativa Nipponbare reference genome using next generation sequence and optical map data," Rice, vol. 6, article 4, 2013.

[15] T. Nagai and A. Makino, "Differences between rice and wheat in temperature responses of photosynthesis and plant growth," Plant and Cell Physiology, vol. 50, no. 4, pp. 744-755, 2009.

[16] Y. Xiang, N. Tang, H. Du, H. Ye, and L. Xiong, "Characterization of OsbZIP23 as a key player of the basic leucine zipper transcription factor family for conferring abscisic acid sensitivity and salinity and drought tolerance in rice," Plant Physiology, vol. 148, no. 4, pp. 1938-1952, 2008.

[17] N. Tang, H. Zhang, X. Li, J. Xiao, and L. Xiong, "Constitutive activation of transcription factor OsbZIP46 improves drought tolerance in rice," Plant Physiology, vol. 158, no. 4, pp. 1755-1768, 2012.

[18] Y. Cao, Q. Zhang, Y. Chen et al., "Identification of differential expression genes in leaves of rice (Oryza sativa L.) in response to heat stress by cDNA-AFLP analysis," BioMed Research International, vol. 2013, Article ID 576189, 11 pages, 2013.

[19] H. Chauhan, N. Khurana, P. Agarwal, and P. Khurana, "Heat shock factors in rice (Oryza sativa L.): genome-wide expression analysis during reproductive development and abiotic stress," Molecular Genetics and Genomics, vol. 286, no. 2, pp. 171-187, 2011.

[20] J. Zou, A. Liu, X. Chen et al., "Expression analysis of nine rice heat shock protein genes under abiotic stresses and ABA treatment," Journal of Plant Physiology, vol. 166, no. 8, pp. 851861, 2009.

[21] D. Mittal, D. A. Madhyastha, and A. Grover, "Gene expression analysis in response to low and high temperature and oxidative stresses in rice: combination of stresses evokes different transcriptional changes as against stresses applied individually," Plant Science, vol. 197, pp. 102-113, 2012.

[22] D. Mittal, D. A. Madhyastha, and A. Grover, "Genome-wide transcriptional profiles during temperature and oxidative stress reveal coordinated expression patterns and overlapping regulons in rice," PLoS ONE, vol. 7, no. 7, Article ID e40899, 2012.

[23] R. B. Saad, D. Fabre, D. Mieulet et al., "Expression of the Aeluropus littoralis AlSAP gene in rice confers broad tolerance to abiotic stresses through maintenance of photosynthesis," Plant, Cell and Environment, vol. 35, no. 3, pp. 626-643, 2012.

[24] M. R. Park, K. Y. Yun, B. Mohanty et al., "Supra-optimal expression of the cold-regulated OsMyb4 transcription factor in transgenic rice changes the complexity of transcriptional network with major effects on stress tolerance and panicle development," Plant, Cell and Environment, vol. 33, no. 12, pp. 2209-2230, 2010.

[25] G. Zhang, G. Guo, X. Hu et al., "Deep RNA sequencing at single base-pair resolution reveals high complexity of the rice transcriptome," Genome Research, vol. 20, no. 5, pp. 646-654, 2010.
[26] T. Lu, G. Lu, D. Fan et al., "Function annotation of the rice transcriptome at single-nucleotide resolution by RNA-seq," Genome Research, vol. 20, no. 9, pp. 1238-1249, 2010.

[27] L. Gu and R. Guo, "Genome-wide detection and analysis of alternative splicing for nucleotide binding site-leucine-rich repeats sequences in rice," Journal of Genetics and Genomics, vol. 34, no. 3, pp. 247-257, 2007.

[28] B. B. Wang and V. Brendel, "Genomewide comparative analysis of alternative splicing in plants," Proceedings of the National Academy of Sciences of the United States of America, vol. 103, no. 18, pp. 7175-7180, 2006. 

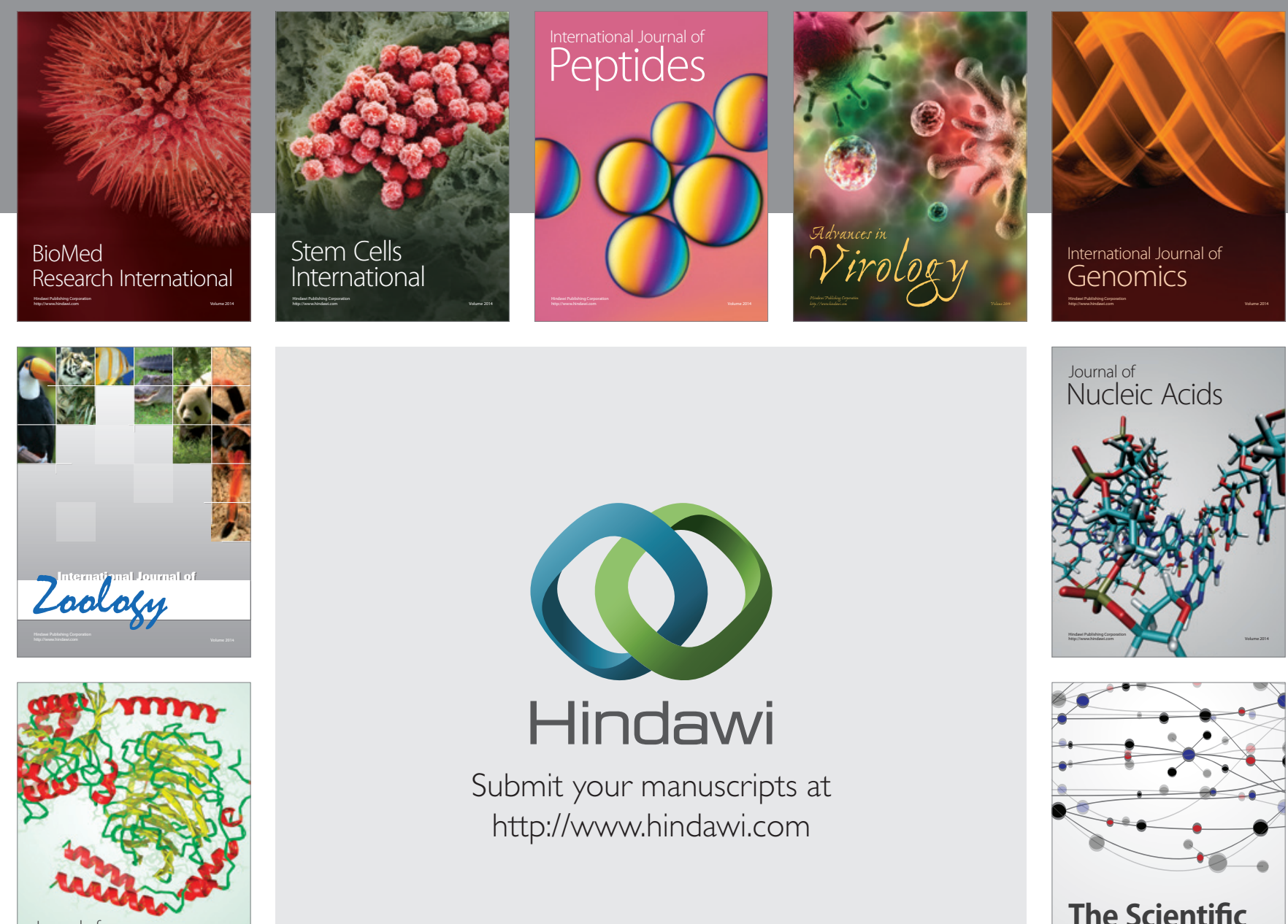

Submit your manuscripts at

http://www.hindawi.com

Journal of
Signal Transduction
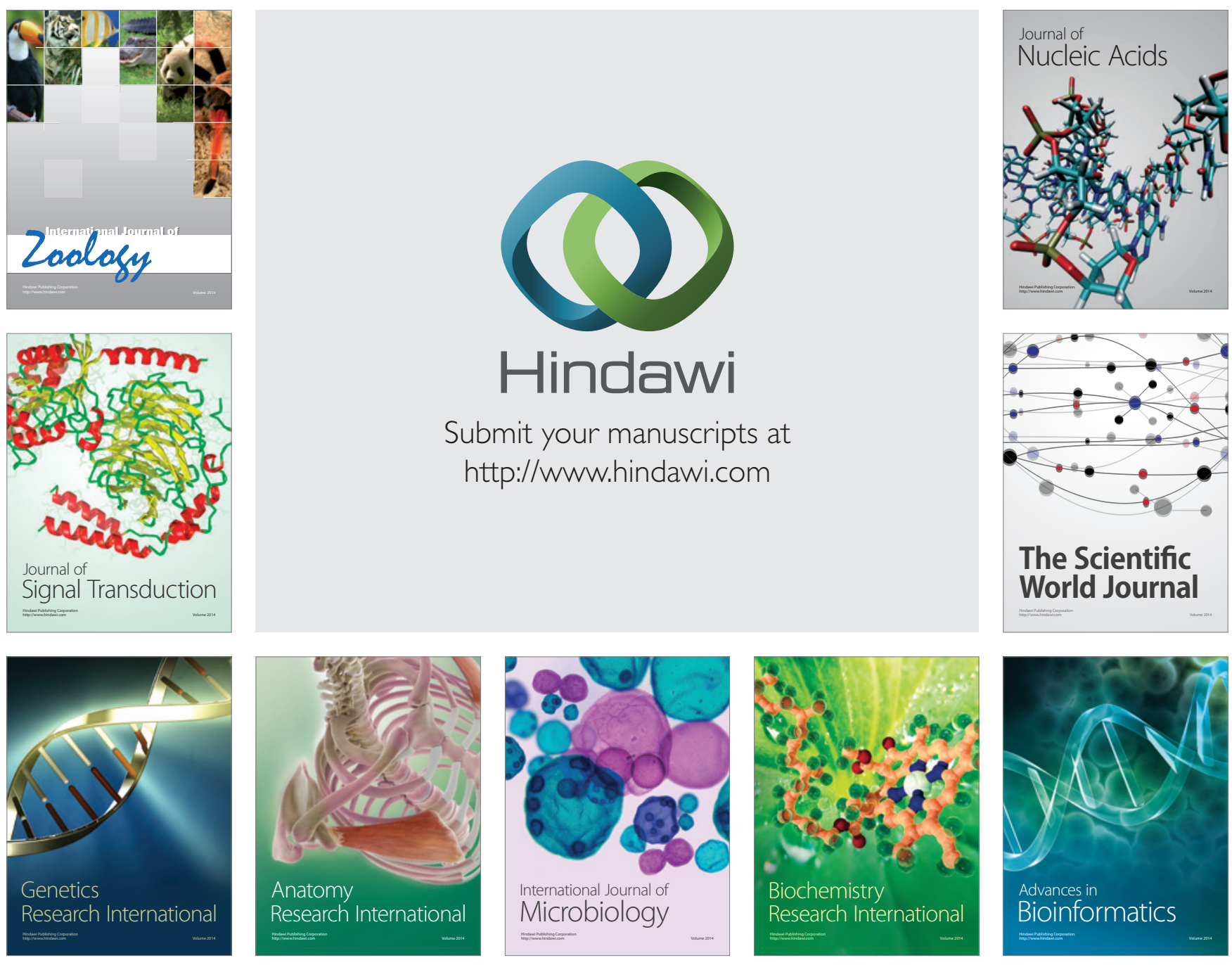

The Scientific World Journal
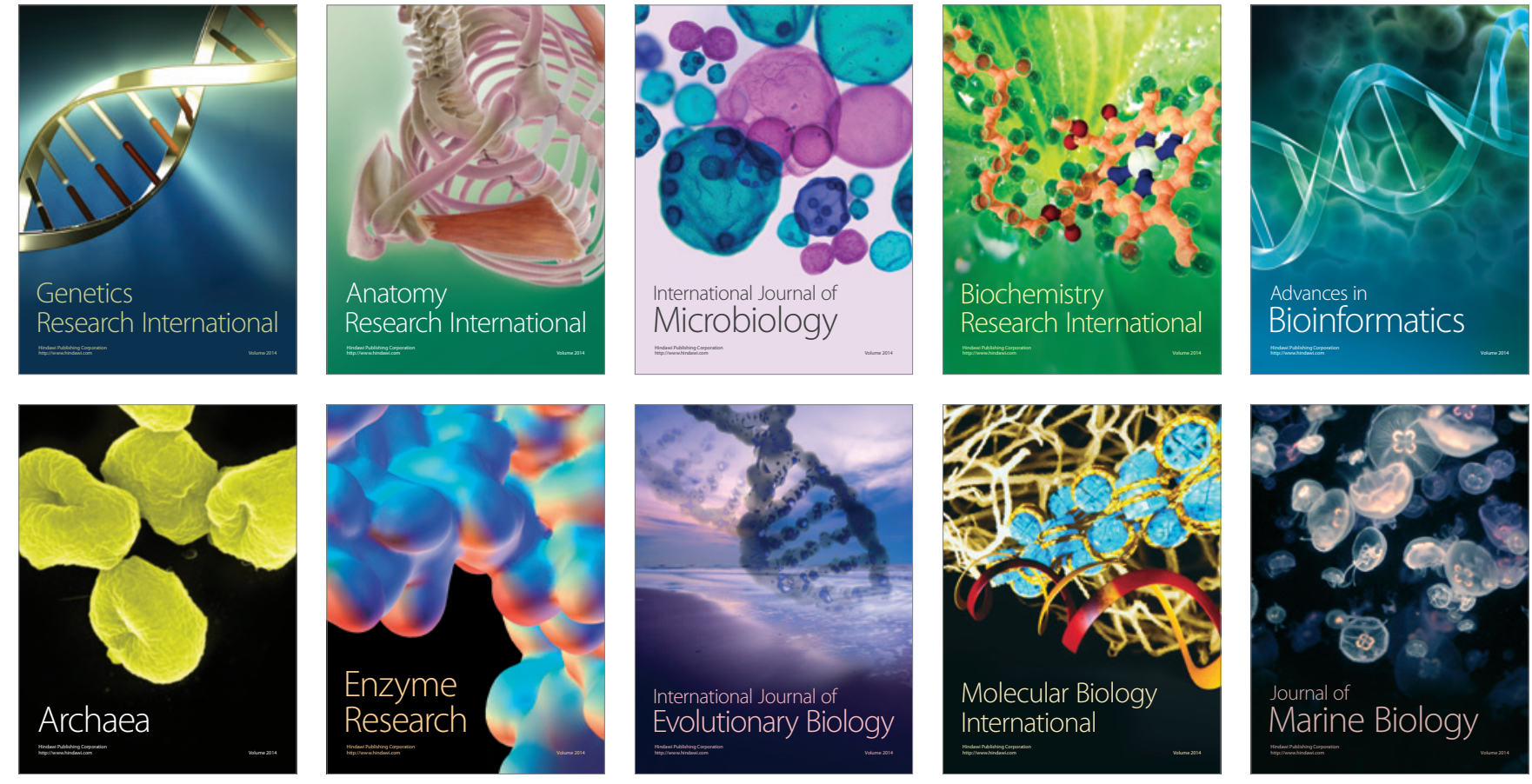